\title{
BOUNDARY VALUE PROBLEMS FOR FUNCTIONAL DIFFERENCE EQUATIONS ON INFINITE INTERVALS
}

\author{
MAURO MARINI, SERENA MATUCCI, AND PAVEL ŘEHÁK
}

Received 27 May 2005; Accepted 29 June 2005

A general method for solving boundary value problems associated to functional difference systems on the discrete half-line is presented and applied in studying the existence of positive unbounded solutions for a system of two coupled nonlinear difference equations. A further example, illustrating the method, completes the paper.

Copyright (C) 2006 Mauro Marini et al. This is an open access article distributed under the Creative Commons Attribution License, which permits unrestricted use, distribution, and reproduction in any medium, provided the original work is properly cited.

\section{Introduction}

A method for solving discrete functional boundary value problems (FBVPs) on infinite intervals is presented and applied to study the existence of positive unbounded solutions of the coupled nonlinear difference system

$$
\begin{aligned}
& \Delta\left(r_{k} \Phi_{\alpha}\left(\Delta x_{k}\right)\right)=-f\left(k, y_{k+1}\right), \\
& \Delta\left(q_{k} \Phi_{\beta}\left(\Delta y_{k}\right)\right)=g\left(k, x_{k+1}\right),
\end{aligned}
$$

where $\Delta$ is the forward difference operator, $r=\left\{r_{k}\right\}, q=\left\{q_{k}\right\}$ are positive real sequences, $\Phi_{\lambda}(u)=|u|^{\lambda-1} \operatorname{sgn} u$ with $\lambda>1$, and $f, g$ are real continuous functions on $\mathbb{N} \times \mathbb{R}$, satisfying additional assumptions that will be specified later. The sequences $r, q$ are assumed to satisfy

$$
\sum_{k=1}^{\infty} \frac{1}{\Phi_{\alpha^{*}}\left(r_{k}\right)}=\infty, \quad \sum_{k=1}^{\infty} \frac{1}{\Phi_{\beta^{*}}\left(q_{k}\right)}=\infty,
$$

where $\alpha^{*}$ and $\beta^{*}$ denote the conjugate numbers of $\alpha$ and $\beta$, respectively, that is, $1 / \alpha+$ $1 / \alpha^{*}=1$ and $1 / \beta+1 / \beta^{*}=1$.

In the last years, an increasing interest has been devoted to investigate the qualitative properties of higher order difference equations and, in particular, fourth order equations. 
They naturally appear in the discretization of a variety of physical, biological and chemical phenomena, such as, for instance, problems of elasticity, deformation of structures or soil settlement (see, e.g., $[9,10]$ ). We refer, for instance, to $[12,14,17-19]$, to the monographs $[3,5]$, and references therein. In particular, in [12] conditions for all the solutions of (1.1) to be oscillatory are presented. Here the existence of positive unbounded solutions of (1.1) is examined: these solutions are classified according to their growth at infinity and necessary and sufficient existence results are obtained. Such results are strictly related also to the recent ones in [14], in which the asymptotic behavior of nonoscillatory solutions of a fourth order nonlinear difference equation is considered.

Our main tool is based on an existence result concerning the solvability of functional boundary value problems on unbounded domains and is presented in the next section. Such a result originates from an existing one stated for differential systems in [8, Theorem 1.2]. By means of this approach, the study of the topological properties (compactness and continuity) of the fixed-point operator, can be quite simplified because, very often, these properties become an immediate consequence of good $a$-priori bounds. Other advantages of our approach are illustrated in Section 2. Applications and examples are given in Sections 3 and 4, respectively.

\section{A fixed point approach}

Let $m \in \mathbb{N}, \mathbb{N}_{m}=\{k \in \mathbb{N}, k \geq m\}$, and denote with $\mathbb{F}$ the Fréchet space of all real sequences defined on $\mathbb{N}_{m}$ endowed with the topology of uniform convergence on compact subsets of $\mathbb{N}_{m}$. We recall that a subset $W \subset \mathbb{F}$ is bounded if and only if it consists of sequences which are equibounded on the discrete interval $[m, m+p]$ for each $p \in \mathbb{N}$, that is, if and only if there exists a sequence $z \in \mathbb{F}$ such that $\left|w_{k}\right| \leq z_{k}$ for each $k \in \mathbb{N}_{m}$ and $w \in W$. Moreover Ascoli theorem implies that any bounded set in $\mathbb{F}$ is relatively compact (see, e.g., [3, Theorem 5.6.1]). Further, let $\mathbb{F}^{n}$ be the Fréchet space of all $n$-vector sequences endowed with the topology induced by the Cartesian product. A vector sequence in $\mathbb{F}^{n}$ will be represented by $\underline{\mathbf{x}}$ and its elements by $\underline{\mathbf{x}}_{k}$. Consider the FBVP

$$
\Delta \underline{\mathbf{x}}_{k}=F\left(k, \underline{\mathbf{x}}_{k}, \underline{\mathbf{x}}\right), \quad k \in \mathbb{N}_{m}, \underline{\mathbf{x}} \in B
$$

where $F: \mathbb{N}_{m} \times \mathbb{R}^{n} \times \mathbb{F}^{n} \rightarrow \mathbb{R}^{n}$ is a continuous map, and $B$ is a subset of $\mathbb{F}^{n}$.

In the last years, FBVPs have attracted considerable attention, both in the continuous and in the discrete case, especially when they are examined on unbounded domains (see, e.g., $[2-4,6,13,16])$. Indeed the functional dependence of the function $F$ in (2.1) allows to treat in a similar way a wide class of boundary value problems, such as the ones associated to advanced, or delayed difference equations, or sum difference equations.

Several approaches can be used in order to treat boundary value problems on infinite intervals; besides the classical ones, such as, for instance, the Schauder (or SchauderTychonoff) fixed point theorem, recently new methods have been proposed, especially as an extension of the Leray-Schauder continuation principle. The reader can refer to the monograph [3] for a good survey on this topic. Here we present a new approach, based on a result stated for the continuous case in [8, Theorem 1.2]. The following holds. 
Theorem 2.1. Let $G: \mathbb{N}_{m} \times \mathbb{R}^{2 n} \times \mathbb{F}^{2 n} \rightarrow \mathbb{R}^{n}$ be a continuous map such that, for $(k, \underline{\mathbf{u}}) \in$ $\mathbb{N}_{m} \times \mathbb{F}^{n}$

$$
G\left(k, \underline{\mathbf{u}}_{k}, \underline{\mathbf{u}}_{k}, \underline{\mathbf{u}}, \underline{\mathbf{u}}\right)=F\left(k, \underline{\mathbf{u}}_{k}, \underline{\mathbf{u}}\right)
$$

If there exists a nonempty, closed, convex and bounded set $\Omega \subset \mathbb{F}^{n}$ such that:

(a) for any $\mathbf{q} \in \Omega$, the problem

$$
\Delta \underline{\mathbf{y}}_{k}=G\left(k, \underline{\mathbf{y}}_{k}, \underline{\mathbf{q}}_{k}, \underline{\mathbf{y}}, \underline{\mathbf{q}}\right), \quad \underline{\mathbf{y}} \in B
$$

has a unique solution $\underline{\mathbf{y}}=T(\underline{\mathbf{q}})$;

(b) $T(\Omega) \subset \Omega$;

(c) $\overline{T(\Omega)} \subset B$;

then (2.1) has at least one solution.

Proof. The argument is similar to that given for the continuous case in [8, Theorem 1.2], with minor changes. For the sake of completeness we briefly sketch the proof.

Let us show that the operator $T: \Omega \rightarrow \Omega$ is continuous with relatively compact image. The relatively compactness of $T(\Omega)$ follows immediately from (b), since $\Omega$ is bounded. To prove the continuity of $T$ in $\Omega$, let $\left\{\underline{\mathbf{q}}^{j}\right\}$ be a sequence in $\Omega, \underline{\mathbf{q}}^{j} \rightarrow \underline{\mathbf{q}}^{\infty} \in \Omega$, and let $\underline{\mathbf{v}}^{j}=T\left(\underline{\mathbf{q}}^{j}\right)$. Since $T(\Omega)$ is relatively compact, $\left\{\underline{\mathbf{v}}^{j}\right\}$ admits a subsequence (still indicated with $\left\{\underline{\mathbf{v}}^{j}\right\}$ to avoid double indexes) which is convergent to $\underline{\mathbf{v}}^{\infty} \in \mathbb{F}^{n}$. In view of (c), we have $\underline{\mathbf{v}}^{\infty} \in B$. Since $G$ is continuous, we obtain

$$
\Delta \underline{\mathbf{v}}_{k}^{\infty}=\lim _{j} \Delta \underline{\mathbf{v}}_{k}^{j}=\lim _{j} G\left(k, \underline{\mathbf{v}}_{k}^{j}, \underline{\mathbf{q}}_{k}^{j}, \underline{\mathbf{v}}^{j}, \underline{\mathbf{q}}^{j}\right)=G\left(k, \underline{\mathbf{v}}_{k}^{\infty}, \underline{\mathbf{q}}_{k}^{\infty}, \underline{\mathbf{v}}^{\infty}, \underline{\mathbf{q}}^{\infty}\right) .
$$

The uniqueness of the solution of (2.3) yields $\underline{\mathbf{v}}^{\infty}=T\left(\mathbf{q}^{\infty}\right)$, and therefore $T$ is continuous on $\Omega$. By the Schauder-Tychonoff fixed point theorem, $T$ has at least one fixed point in $\Omega$, which is a solution of (2.1), as it can be easily checked.

Remark 2.2. Analogously to the continuous case, as follows from the proof of Theorem 2.1 , the operator $T$, defined by condition (a), has a relatively compact image provided that condition (b) holds. If, moreover, the closure $\overline{T(\Omega)}$ is contained in $B$, then $T$ is also continuous in $\Omega$. In practice, these conditions may be directly derived by the existence of appropriate a-priori bounds on the solutions of (2.3). So the map $G$ has to be wellchosen, and an optimal choice can be made by taking a map $G$ which is linear with respect to the second variable, and does not depend on the fourth one. From this point of view, our approach is very similar to the Schauder linearization device, as the applications and examples in the subsequent sections will illustrate.

Remark 2.3. In dealing with boundary value problems on infinite intervals, the use of the Fréchet space $\mathbb{F}$ has some advantages over the use of a suitable Banach space due to compactness test. Indeed, as claimed, a subset $W \subset \mathbb{F}$ is relatively compact in $\mathbb{F}$ if it is bounded in $\mathbb{F}$ and this condition can be easily checked, as the subsequent applications will show. Moreover, seeking a Banach space, the compactness test may not be easy to check. For 
instance, in the Banach space $\ell^{\infty}$ of all bounded real sequences, the compactness test of a subset $W \subset \ell^{\infty}$ requires to verify, besides the boundedness, some additional properties (see, e.g., [3, Remark 5.3.1]), that may be difficult to check, for instance, when sequences in $W$ do not admit a limit as $k \rightarrow \infty$. In addition, if the Banach space is a weighted space, that is, $\ell_{w}^{\infty}=\left\{u: \sup _{k}\left|u_{k} / w_{k}\right|<\infty\right\}$, being $w$ a positive fixed sequence, then the proof of the compactness may be even less immediate. Notice that, to work in a Banach space, a weighted space has to be chosen for solving boundary value problems related to existence of unbounded solutions.

Remark 2.4. If $B$ is closed, as it often happens for boundary value problems on finite discrete intervals, then condition (c) is trivially satisfied. If the interval, in which the problem has to be considered, is infinite, and the boundary conditions involve the behavior of the solution at infinity, then $B$ may not be closed. A weaker condition than (c) is

(c1) if $\left\{\underline{\mathbf{q}}^{m}\right\}$ is a sequence in $\Omega$ converging in $\Omega$ and $T\left(\underline{\mathbf{q}}^{m}\right) \rightarrow \underline{\mathbf{q}}^{\infty}$ (in the topology of $\left.\mathbb{F}^{n}\right)$, then $\underline{\mathbf{q}}^{\underline{\infty}} \in B$.

In particular, if conditions (a) and (b) are satisfied, then it is easy to verify that (c1) becomes also necessary for the continuity of $T$ in $\Omega$.

Remark 2.5. The functional dependence can also appear when the solvability of a boundary value problem is accomplished by means of a suitable change of variables, which reduces higher order difference equations to functional difference equations of lower order. For instance, given a second order equation in the unknown $x$, the change of variable $w_{k}=\Delta x_{k}$ gives $x_{k}=\sum_{j=1}^{k-1} w_{k}+x_{1}$, if $x_{1}$ is known, or $x_{k}=x^{\infty}-\sum_{j=k}^{\infty} w_{j}$, if $x^{\infty}=\lim _{k} x_{k}$ is finite. In both cases we have $x_{k}=(S[w])_{k}$, with a clear meaning of the operator $S$. An example of this approach is given in the next section.

In the particular case of FBVPs for scalar difference equations of order $n$

$$
\Delta^{n} x_{k}=\widetilde{F}\left(k, x_{k}, \ldots, x_{k+n-1}, x\right), \quad x \in \widetilde{B},
$$

where $\widetilde{F}: \mathbb{N}_{m} \times \mathbb{R}^{n} \times \mathbb{F} \rightarrow \mathbb{R}$ is a continuous map, and $\widetilde{B}$ is a subset of $\mathbb{F}$, the assumptions of Theorem 2.1 can be slightly simplified, because good a-priori bounds for the unknown $x$ are sufficient to treat FBVPs for a scalar difference equations of higher order. Indeed, in the discrete case, if a set $\widetilde{\Omega} \subset \mathbb{F}$ is bounded, $\widetilde{\Omega}^{\Delta}=\{\Delta u, u \in \widetilde{\Omega}\}$ is bounded, too. The following holds.

Corollary 2.6. Let $\widetilde{G}: \mathbb{N}_{m} \times \mathbb{R}^{2 n} \times \mathbb{F}^{2} \rightarrow \mathbb{R}$ be a continuous map such that, for $(k, u) \in$ $\mathbb{N}_{m} \times \mathbb{F}$

$$
\tilde{G}\left(k, u_{k}, \ldots, u_{k+n-1}, u_{k}, \ldots, u_{k+n-1}, u, u\right)=\tilde{F}\left(k, u_{k}, \ldots, u_{k+n-1}, u\right)
$$

If there exists a nonempty, closed, convex and bounded set $\widetilde{\Omega} \subset \mathbb{F}$ such that:

(a) for any $q \in \widetilde{\Omega}$, the problem

$$
\Delta^{n} y_{k}=\widetilde{G}\left(k, y_{k}, \ldots, y_{k+n-1}, q_{k}, \ldots, q_{k+n-1}, y, q\right), \quad y \in \widetilde{B}
$$

has a unique solution $y=\tilde{T}(q)$; 
(b) $\widetilde{T}(\widetilde{\Omega}) \subset \widetilde{\Omega}$;

(c) $\overline{\widetilde{T}(\widetilde{\Omega})} \subset \widetilde{B}$;

then (2.5) has at least one solution.

Proof. The proof can be easily done, following the same arguments as in the proof of Theorem 2.1, with minor changes.

As a final remark, notice that any difference equation of higher order can always be understood as a first order equation with deviating arguments.

\section{Unbounded solutions of (1.1)}

In this section we study the existence of solutions $(x, y), x=\left\{x_{k}\right\}, y=\left\{y_{k}\right\}$, of (1.1), having both components unbounded. For the sake of simplicity, we will restrict our attention only to unbounded solutions whose components are both eventually positive. The remaining cases can be easily treated using the results of this section and some symmetry arguments. As usually, a component $x[y]$ of a solution $(x, y)$ of $(1.1)$ is said to be nonoscillatory if there exists $v \in \mathbb{N}$ such that $x_{k} x_{k+1}>0\left[y_{k} y_{k+1}>0\right]$ for any $k \in \mathbb{N}, k \geq v$, and oscillatory otherwise.

Assume that $f$ and $g$ satisfy the following additional assumptions: $f$ and $g$ are nondecreasing with respect to the second variable; $f(k, u) u>0, g(k, u) u>0$ for $(k, u) \in \mathbb{N} \times$ $\mathbb{R} \backslash\{0\} ; \forall B>1, \exists C_{f}, C_{g} \geq 1$, depending on $B$, such that

$$
\begin{gathered}
f(k, B u) \leq C_{f} f(k, u), \quad \forall(k, u) \in \mathbb{N}_{m} \times[1, \infty), \\
g(k, B u) \leq C_{g} g(k, u), \quad \forall(k, u) \in \mathbb{N}_{m} \times[1, \infty) .
\end{gathered}
$$

Conditions (H1) and (H2) involve the asymptotic behavior of $f$ and $g$ only for positive values of the second variable. If unbounded solutions with components not both positive are to be considered, then the above assumptions need to be modified consequently. Clearly $f$ satisfies (H1) when any of the following two cases occurs for $(k, u) \in$ $\mathbb{N}_{m} \times[1, \infty):$

( $\left.\mathrm{E}_{1}\right) f(k, u)=\psi_{k} h(u)$, where $\psi$ is a positive sequence and $h$ is a positive function, homogeneous of degree $\gamma>0$, or, more generally, a positive regularly varying function [11],

$\left(\mathrm{E}_{2}\right) \exists \gamma>0$ such that $f(k, u) / u^{\gamma}$ is nonincreasing in $u$.

We start by briefly summarizing some basic properties of solutions of (1.1), which were analyzed in detail in [12]. In view of the sign assumptions on $f$ and $g$, it is easy to show that either $x, y$ are both nonoscillatory or $x, y$ are both oscillatory. Thus a solution $(x, y)$ is said to be oscillatory or nonoscillatory according to $x$ and $y$ are both oscillatory or nonoscillatory. Clearly, if a solution $(x, y)$ of $(1.1)$ is nonoscillatory, then also the quasidifferences $x^{[1]}=\left\{x_{k}^{[1]}\right\}, y^{[1]}=\left\{y_{k}^{[1]}\right\}$, where

$$
x_{k}^{[1]}=r_{k} \Phi_{\alpha}\left(\Delta x_{k}\right), \quad y_{k}^{[1]}=q_{k} \Phi_{\beta}\left(\Delta y_{k}\right),
$$


are both nonoscillatory, and therefore $x$ and $y$ are eventually monotone. The following holds.

LEMma 3.1. Every eventually positive unbounded solution $(x, y)$ of $(1.1)$ belongs to any of the following classes:

(i) $\lim _{k} x_{k}^{[1]}=x_{\infty}^{[1]}=$ const. $>0, \lim _{k} y_{k}^{[1]}=y_{\infty}^{[1]}=$ const. $>0$;

(ii) $\lim _{k} x_{k}^{[1]}=x_{\infty}^{[1]}=0, \lim _{k} y_{k}^{[1]}=y_{\infty}^{[1]}=$ const. $>0$;

(iii) $\lim _{k} x_{k}^{[1]}=x_{\infty}^{[1]}=$ const. $>0, \lim _{k} y_{k}^{[1]}=y_{\infty}^{[1]}=\infty$;

(iv) $\lim _{k} x_{k}^{[1]}=x_{\infty}^{[1]}=0, \lim _{k} y_{k}^{[1]}=y_{\infty}^{[1]}=\infty$.

Proof. Let $(x, y)$ be an eventually positive unbounded solution of (1.1). Then $x^{[1]}$ is eventually decreasing and $y^{[1]}$ is eventually increasing. Since $x, y$ are unbounded, then $x_{\infty}^{[1]} \geq$ $0, y_{\infty}^{[1]}>0$.

Put, for the sake of simplicity, $(1 \leq m<k)$

$$
R_{m, k}:=\sum_{j=m}^{k-1} \Phi_{\alpha *}\left(\frac{1}{r_{j}}\right), \quad Q_{m, k}:=\sum_{j=m}^{k-1} \Phi_{\beta *}\left(\frac{1}{q_{j}}\right) .
$$

In view of (1.2), if an eventually positive solution $(x, y)$ of $(1.1)$ is in the class (i), then there exist two positive constants $L_{x}, L_{y}$ such that

$$
\lim _{k} \frac{x_{k}}{R_{1, k}}=L_{x}, \quad \lim _{k} \frac{y_{k}}{Q_{1, k}}=L_{y}
$$

and vice versa. Similar results hold for the other classes, with $L_{x}=0$ for the classes (ii) and (iv), and $L_{y}=\infty$ for the classes (iii) and (iv).

In what follows we will use a usual convention, namely $\sum_{k=n}^{n-1} a_{k}=0$, for any sequence $a$ and any $n \in \mathbb{N}$.

Concerning the existence of positive unbounded solutions of (1.1) in the class (i), the following holds.

Theorem 3.2. System (1.1) admits eventually positive unbounded solutions belonging to the class (i) if and only if

$$
\sum_{k=1}^{\infty} f\left(k, Q_{1, k+1}\right)<\infty, \quad \sum_{k=1}^{\infty} g\left(k, R_{1, k+1}\right)<\infty .
$$

In addition, if (3.4) is satisfied, then for every couple of positive constants $\left(M_{x}, M_{y}\right)$ there exist infinitely many eventually positive unbounded solutions $(x, y)$ of $(1.1)$ such that $x_{\infty}^{[1]}=$ $M_{x}, y_{\infty}^{[1]}=M_{y}$.

Proof. Let $(x, y)$ be an eventually positive solution of (1.1) in the class (i). In view of (3.3), two positive constants $d_{1}, d_{2}$, and $m \in \mathbb{N}$ exist such that $d_{1} R_{1, k} \leq x_{k}, d_{2} Q_{1, k} \leq y_{k}$, 
for $k \geq m$. By summing (1.1) we have

$$
\begin{gathered}
x_{k+1}^{[1]}-x_{m}^{[1]}=-\sum_{j=m}^{k} f\left(j, y_{j+1}\right) \leq-\sum_{j=m}^{k} f\left(j, d_{2} Q_{1, j+1}\right), \\
y_{k+1}^{[1]}-y_{m}^{[1]}=\sum_{j=m}^{k} g\left(j, x_{j+1}\right) \geq \sum_{j=m}^{k} g\left(j, d_{1} R_{1, j+1}\right) .
\end{gathered}
$$

Since $x^{[1]}$ and $y^{[1]}$ are both convergent, we obtain $\sum_{j=1}^{\infty} f\left(j, d_{2} Q_{1, j+1}\right)<\infty, \sum_{j=1}^{\infty} g(j$, $\left.d_{1} R_{1, j+1}\right)<\infty$. If $d_{2} \geq 1$, then the convergence of the first series in (3.4) follows, since $f$ is nondecreasing with respect to the second variable. On the other hand, if $d_{2}<1$, the assertion comes from (H1), with $B=1 / d_{2}$. The convergence of the second series in (3.4) follows in a similar way.

Conversely, let $M_{x}, M_{y}$ be two positive constants and let $m$ be an integer so large that

$$
\sum_{k=m}^{\infty} f\left(k, \Phi_{\beta *}\left(M_{y}\right) Q_{m, k+1}\right) \leq M_{x}, \quad \sum_{k=m}^{\infty} g\left(k, \Phi_{\alpha *}\left(2 M_{x}\right) R_{m, k+1}\right) \leq \frac{M_{y}}{2} .
$$

Note that (3.6) follows from (3.4), (H1), and (H2). Let $S_{i}: \mathbb{F} \rightarrow \mathbb{F}, i=1,2$, be the operators defined by $S_{1}[w]=\left\{\left(S_{1}[w]\right)_{k}\right\}, S_{2}[z]=\left\{\left(S_{2}[z]\right)_{k}\right\}$, where

$$
\left(S_{1}[w]\right)_{k}=\sum_{j=m}^{k-1} \Phi_{\alpha *}\left(\frac{w_{j}}{r_{j}}\right), \quad\left(S_{2}[z]\right)_{k}=\sum_{j=m}^{k-1} \Phi_{\beta *}\left(\frac{z_{j}}{q_{j}}\right) .
$$

Consider the FBVP

$$
\begin{aligned}
\Delta w_{k} & =-f\left(k,\left(S_{2}[z]\right)_{k+1}\right), \\
\Delta z_{k} & =g\left(k,\left(S_{1}[w]\right)_{k+1}\right), \\
\lim _{k} w_{k} & =M_{x}, \quad \lim _{k} z_{k}=M_{y} .
\end{aligned}
$$

Notice that (3.8) is a functional boundary value problem of the form (2.1) and therefore we can apply Theorem 2.1 to solve it. Let $\Omega \subset \mathbb{F}^{2}$ be the set defined as

$$
\Omega=\left\{(u, v) \in \mathbb{F}^{2}: M_{x} \leq u_{k} \leq 2 M_{x}, \frac{M_{y}}{2} \leq v_{k} \leq M_{y}\right\}
$$

and for every $(u, v) \in \Omega$ consider the linearized boundary value problem

$$
\begin{gathered}
\Delta w_{k}=-f\left(k,\left(S_{2}[v]\right)_{k+1}\right), \\
\Delta z_{k}=g\left(k,\left(S_{1}[u]\right)_{k+1}\right), \\
\lim _{k} w_{k}=M_{x}, \quad \lim _{k} z_{k}=M_{y} .
\end{gathered}
$$


Clearly (3.10) admits a unique solution $(w, z)=T(u, v)$, given by $T(u, v)=\left(T_{1} v, T_{2} u\right)$, where

$$
\left(T_{1} v\right)_{k}=M_{x}+\sum_{j=k}^{\infty} f\left(j,\left(S_{2}[v]\right)_{j+1}\right), \quad\left(T_{2} u\right)_{k}=M_{y}-\sum_{j=k}^{\infty} g\left(j,\left(S_{1}[u]\right)_{j+1}\right) .
$$

The map $T$ is well defined in $\Omega$, and for $k \geq m \geq 1$ we have

$$
\begin{aligned}
& \sum_{j=k}^{\infty} f\left(j,\left(S_{2}[v]\right)_{j+1}\right) \leq \sum_{j=m}^{\infty} f\left(j, \Phi_{\beta *}\left(M_{y}\right) Q_{m, j+1}\right) \leq M_{x}, \\
& \sum_{j=k}^{\infty} g\left(j,\left(S_{1}[u]\right)_{j+1}\right) \leq \sum_{j=m}^{\infty} g\left(j, \Phi_{\alpha *}\left(2 M_{x}\right) R_{m, j+1}\right) \leq \frac{M_{y}}{2} .
\end{aligned}
$$

Therefore $T(\Omega) \subseteq \Omega$. The proof that condition (c) of Theorem 2.1 is satisfied, with

$$
B=\left\{(w, z) \in \mathbb{F}^{2}: \lim _{k} w_{k}=M_{x}, \lim _{k} z_{k}=M_{y}\right\}
$$

is an easy consequence of the discrete dominated convergence theorem, whose applicability is guaranteed by the estimates (3.12). Indeed, let $\left\{\left(T_{1} v^{n}, T_{2} u^{n}\right)\right\}$ be a sequence in $T(\Omega)$, converging to $(\hat{w}, \hat{z})$ in $\mathbb{F}^{2}$. Since $\Omega$ is compact, we can assume that the sequence $\left\{\left(u^{n}, v^{n}\right)\right\} \subset \Omega$ converges to $(\hat{u}, \hat{v})$ in $\Omega$. Then the continuity of $f, g$ and $S_{i}, i=1,2$ yields $\lim _{n} f\left(j,\left(S_{2}\left[v^{n}\right]\right)_{j+1}\right)=f\left(j,\left(S_{2}[\hat{v}]\right)_{j+1}\right), \lim _{n} g\left(j,\left(S_{1}\left[u^{n}\right]\right)_{j+1}\right)=g\left(j,\left(S_{1}[\hat{u}]\right)_{j+1}\right)$, for all $j \geq m$. Since $(\hat{u}, \hat{v}) \in \Omega$, and the estimates (3.12) hold, the dominated convergence theorem leads to $(\hat{w}, \hat{z})=\lim _{n}\left(T_{1} v^{n}, T_{2} u^{n}\right)=\left(T_{1} \hat{v}, T_{2} \hat{u}\right) \in B$. Theorem 2.1 can be therefore applied to problem (3.8), obtaining the existence of at least one solution. Let $(\bar{w}, \bar{z})$ be such a solution; clearly $(\bar{x}, \bar{y})=\left(S_{1}[\bar{w}], S_{2}[\bar{z}]\right)$ is a solution of (1.1) in the class (i), with $\bar{x}_{m}=\bar{y}_{m}=0$. Finally the existence of infinitely many solutions in the class (i) follows by using the same argument, with minor changes. Instead of (3.7) and (3.6) it is sufficient to consider

$$
\begin{gathered}
\left(S_{1}[w]\right)_{k}=a_{1}+\sum_{j=m}^{k-1} \Phi_{\alpha *}\left(\frac{w_{j}}{r_{j}}\right), \quad\left(S_{2}[z]\right)_{k}=a_{2}+\sum_{j=m}^{k-1} \Phi_{\beta *}\left(\frac{z_{j}}{q_{j}}\right), \\
\sum_{k=m}^{\infty} f\left(k, a_{2}+\Phi_{\beta *}\left(M_{y}\right) Q_{m, k+1}\right) \leq M_{x}, \quad \sum_{k=m}^{\infty} g\left(k, a_{1}+\Phi_{\alpha *}\left(2 M_{x}\right) R_{m, k+1}\right) \leq \frac{M_{y}}{2},
\end{gathered}
$$

respectively, where $a_{1}, a_{2}$ are two arbitrarily positive constants. In this case, we obtain a solution $(\bar{x}, \bar{y})=\left(S_{1}[\bar{w}], S_{2}[\bar{z}]\right)$ of $(1.1)$ belonging to the class (i), with $\bar{x}_{m}=a_{1}, \bar{y}_{m}=$ $a_{2}$.

As follows from the proof of Theorem 3.2, the used change of variables decreases the order of the system. It is transformed into a first order system, but of functional type, and the application of our existence theorem (Theorem 2.1) leads to easier subsequent computations. 
Concerning solutions in the class (ii), the following result holds. Its proof is similar, with minor changes, to the one of Theorem 3.2.

Theorem 3.3. System (1.1) admits eventually positive unbounded solutions belonging to the class (ii) if and only if

$$
\begin{gathered}
\sum_{k=1}^{\infty} \Phi_{\alpha^{*}}\left(\frac{1}{r_{k}} \sum_{j=k}^{\infty} f\left(j, Q_{1, j+1}\right)\right)=\infty, \\
\sum_{k=1}^{\infty} g\left(k, \sum_{j=1}^{k} \Phi_{\alpha^{*}}\left(\frac{1}{r_{j}} \sum_{i=j}^{\infty} f\left(i, Q_{1, i+1}\right)\right)\right)<\infty .
\end{gathered}
$$

In addition, if (3.15) is satisfied, then for every positive constant $M_{y}$ there exist infinitely many eventually positive unbounded solutions $(x, y)$ of $(1.1)$ such that $x_{\infty}^{[1]}=0, y_{\infty}^{[1]}=M_{y}$.

The existence of solutions in the classes (iii) and (iv) is considered in the subsequent two theorems. Since, in both cases, $y^{[1]}$ is unbounded, the change of variables that leads to a first order system is now different from the previous cases.

Theorem 3.4. System (1.1) admits eventually positive unbounded solutions belonging to the class (iii) if and only if

$$
\begin{gathered}
\sum_{k=1}^{\infty} g\left(k, R_{1, k+1}\right)=\infty, \\
\sum_{k=1}^{\infty} f\left(k, \sum_{j=1}^{k} \Phi_{\beta^{*}}\left(\frac{1}{q_{j}} \sum_{i=1}^{j-1} g\left(i, R_{1, i+1}\right)\right)\right)<\infty .
\end{gathered}
$$

In addition, if (3.16) is satisfied, then for every positive constant $M_{x}$ there exist infinitely many eventually positive unbounded solutions of (1.1) such that $x_{\infty}^{[1]}=M_{x}, y_{\infty}^{[1]}=\infty$.

Proof. Let $(x, y)$ be a solution of (1.1) in the class (iii). Then two positive constants $d_{1} \leq d_{2}$ exist such that $d_{1} R_{1, k} \leq x_{k} \leq d_{2} R_{1, k}$, for $k \geq m \geq 1$, where $m$ is sufficiently large. We can assume $d_{1} \leq 1, d_{2} \geq 1$. By summing the second equation in (1.1), we obtain

$$
\sum_{j=m}^{k} g\left(j, d_{1} R_{1, j+1}\right) \leq y_{k+1}^{[1]}-y_{m}^{[1]} \leq \sum_{j=m}^{k} g\left(j, d_{2} R_{1, j+1}\right),
$$

and the divergence of the first series in (3.16) follows, since $y_{\infty}^{[1]}=\infty, d_{2} \geq 1$, and $g$ satisfies (H2). From (3.17) we have

$$
y_{k}^{[1]} \geq \sum_{j=m}^{k-1} g\left(j, d_{1} R_{1, j+1}\right),
$$


which implies

$$
y_{k+1} \geq \sum_{j=m}^{k} \Phi_{\beta^{*}}\left(\frac{1}{q_{j}} \sum_{i=m}^{j-1} g\left(i, d_{1} R_{1, i+1}\right)\right) .
$$

By summing the first equation in (1.1), from (3.19) we obtain

$$
x_{k+1}^{[1]}-x_{m}^{[1]} \leq-\sum_{j=m}^{k} f\left(j, \sum_{i=m}^{j} \Phi_{\beta^{*}}\left(\frac{1}{q_{i}} \sum_{n=m}^{i-1} g\left(n, d_{1} R_{1, n+1}\right)\right)\right) .
$$

Since $g$ satisfies (H2), and $1 / d_{1} \geq 1$, we get $g\left(n, d_{1} R_{1, n+1}\right) \geq g\left(r, R_{1, n+1}\right) / C_{1}$ for a suitable $C_{1} \geq 1$. Further, since $f$ satisfies (H1), we get the existence of a constant $C_{2} \geq 1$ such that

$$
f\left(j, \sum_{i=m}^{j} \Phi_{\beta^{*}}\left(\frac{1}{C_{1} q_{i}} \sum_{n=m}^{i-1} g\left(n, R_{1, n+1}\right)\right)\right) \geq \frac{1}{C_{2}} f\left(j, \sum_{i=m}^{j} \Phi_{\beta^{*}}\left(\frac{1}{q_{i}} \sum_{n=m}^{i-1} g\left(n, R_{1, n+1}\right)\right)\right) .
$$

The convergence of the second series in (3.16) now follows, taking into account that $x^{[1]}$ has a finite limit.

Conversely, let $M_{x}>0$ be a fixed constant and let $m$ be a sufficiently large integer such that

$$
\sum_{k=m}^{\infty} f\left(k, \sum_{j=m}^{k} \Phi_{\beta *}\left(\frac{1}{q_{j}} \sum_{i=m}^{j-1} g\left(i, \Phi_{\alpha *}\left(2 M_{x}\right) R_{m, i+1}\right)\right)\right) \leq M_{x}
$$

Notice that the convergence of the second series in (3.16) assures that (3.22) is well posed, taking into account that $f$ and $g$ are nondecreasing and satisfy (H1) and (H2), respectively. Let $S_{i}: \mathbb{F} \rightarrow \mathbb{F}, i=1,2$, be the operators given by (3.7), and consider the FBVP

$$
\begin{gathered}
\Delta w_{k}=-f\left(k,\left(S_{2}[z]\right)_{k+1}\right), \\
\Delta z_{k}=g\left(k,\left(S_{1}[w]\right)_{k+1}\right), \\
\lim _{k} w_{k}=M_{x}, \quad z_{m}=0 .
\end{gathered}
$$

Let $\Omega \subset \mathbb{F}^{2}$ be the set

$$
\begin{gathered}
\Omega=\left\{(u, v) \in \mathbb{F}^{2}: M_{x} \leq u_{k} \leq 2 M_{x}, \sum_{j=m}^{k-1} g\left(j, \Phi_{\alpha^{*}}\left(M_{x}\right) R_{m, j+1}\right) \leq v_{k}\right. \\
\left.\leq \sum_{j=m}^{k-1} g\left(j, \Phi_{\alpha^{*}}\left(2 M_{x}\right) R_{m, j+1}\right)\right\}
\end{gathered}
$$

and for every $(u, v) \in \Omega$ consider the linearized problem

$$
\begin{gathered}
\Delta w_{k}=-f\left(k,\left(S_{2}[v]\right)_{k+1}\right), \\
\Delta z_{k}=g\left(k,\left(S_{1}[u]\right)_{k+1}\right), \\
\lim _{k} w_{k}=M_{x}, \quad z_{m}=0 .
\end{gathered}
$$


Clearly (3.25) admits a unique solution $(w, z)=T(u, v)$, given by $T(u, v)=\left(T_{1} v, T_{2} u\right)$, with

$$
\left(T_{1} v\right)_{k}=M_{x}+\sum_{j=k}^{\infty} f\left(j,\left(S_{2}[v]\right)_{j+1}\right), \quad\left(T_{2} u\right)_{k}=\sum_{j=m}^{k-1} g\left(j,\left(S_{1}[u]\right)_{j+1}\right) .
$$

The map $T$ is well defined in $\Omega$, and for $k \geq m \geq 1$ we have

$$
\begin{aligned}
& \sum_{j=k}^{\infty} f\left(j,\left(S_{2}[v]\right)_{j+1}\right) \leq \sum_{j=m}^{\infty} f\left(j, \sum_{i=m}^{k} \Phi_{\beta *}\left(\frac{1}{q_{i}} \sum_{n=m}^{i-1} g\left(n, \Phi_{\alpha *}\left(2 M_{x}\right) R_{m, n+1}\right)\right)\right) \leq M_{x} \\
& \sum_{j=m}^{k-1} g\left(j, \Phi_{\alpha *}\left(M_{x}\right) R_{m, j+1}\right) \leq \sum_{j=m}^{k-1} g\left(j,\left(S_{1}[u]\right)_{j+1}\right) \leq \sum_{j=m}^{k-1} g\left(j, \Phi_{\alpha *}\left(2 M_{x}\right) R_{m, j+1}\right) .
\end{aligned}
$$

Then $T(\Omega) \subseteq \Omega$. Further, the above estimates and the discrete dominated convergence theorem also assure that condition (c) of Theorem 2.1 is satisfied, where $B=\{(w, z) \in$ $\left.\mathbb{F}^{2}: \lim _{k} w_{k}=M_{x}, z_{m}=0\right\}$. Therefore (3.23) has at least one solution $(\bar{w}, \bar{z})$, and clearly $(\bar{x}, \bar{y})=\left(S_{1}[\bar{w}], S_{2}[\bar{z}]\right)$ is a solution of (1.1) in the class (iii), with $\bar{x}_{m}=\bar{y}_{m}=\bar{y}_{m}^{[1]}=0$. Finally, the existence of infinitely many solutions in the class (iii) follows by using the same argument, with minor changes. Instead of (3.7) and (3.22) it is sufficient to consider

$$
\begin{gathered}
\left(S_{1}[w]\right)_{k}=a+\sum_{j=m}^{k-1} \Phi_{\alpha *}\left(\frac{w_{j}}{r_{j}}\right), \quad\left(S_{2}[z]\right)_{k}=\sum_{j=m}^{k-1} \Phi_{\beta *}\left(\frac{z_{j}}{q_{j}}\right), \\
\sum_{k=m}^{\infty} f\left(k, \sum_{j=m}^{k} \Phi_{\beta *}\left(\frac{b}{q_{j}}+\frac{1}{q_{j}} \sum_{i=m}^{j-1} g\left(i, a+\Phi_{\alpha *}\left(2 M_{x}\right) R_{m, i+1}\right)\right)\right) \leq M_{x},
\end{gathered}
$$

respectively, where $a, b$ are two arbitrarily positive constants. The boundary value problem to be solved is the system in (3.23) with the conditions $\lim _{k} w_{k}^{[1]}=0, z_{m}=b$; it has at least one solution $(\bar{w}, \bar{z})$ in the set

$$
\begin{aligned}
\Omega=\{( & (u, v) \in \mathbb{F}^{2}: M_{x} \leq u_{k} \leq 2 M_{x}, \sum_{j=m}^{k-1} g\left(j, \Phi_{\alpha^{*}}\left(M_{x}\right) R_{m, j+1}\right) \leq v_{k}-b \\
& \left.\leq \sum_{j=m}^{k-1} g\left(j, a+\Phi_{\alpha^{*}}\left(2 M_{x}\right) R_{m, j+1}\right)\right\} .
\end{aligned}
$$

In this case, we obtain a solution $(\bar{x}, \bar{y})=\left(S_{1}[\bar{w}], S_{2}[\bar{z}]\right)$ of (1.1) belonging to the class (iii), with $\bar{x}_{m}=a, \bar{y}_{m}=0, \bar{y}_{m}^{[1]}=b$.

Notice that Theorem 3.4 extends [14, Theorem 2.6]. Concerning the existence in the class (iv), a sufficient condition is given in the following result, which can be proved by using a similar argument as that given in the proof of Theorem 3.4. 
THeOrem 3.5. If

$$
\begin{gathered}
\sum_{k=1}^{\infty} g(k, 1)=\infty \\
\sum_{k=1}^{\infty} f\left(k, \sum_{j=1}^{k} \Phi_{\beta^{*}}\left(\frac{1}{q_{j}} \sum_{i=1}^{j-1} g\left(i, R_{1, i+1}\right)\right)\right)<\infty \\
\sum_{k=1}^{\infty} \Phi_{\alpha^{*}}\left(\frac{1}{r_{k}} \sum_{j=k}^{\infty} f\left(j, \sum_{i=1}^{j} \Phi_{\beta^{*}}\left(\frac{1}{q_{i}} \sum_{\ell=1}^{i-1} g(\ell, 1)\right)\right)\right)=\infty,
\end{gathered}
$$

then there exist infinitely many positive unbounded solutions of (1.1) such that $x_{\infty}^{[1]}=0$, $y_{\infty}^{[1]}=\infty$.

\section{A further example}

Here we present a further example illustrating the role of function $G$ in Theorem 2.1. It is well-known ([1, Theorems 6.10.4 and 6.11.1]; see also [7, Theorem 5]), that the equation

$$
\Delta^{2} x_{k}=s_{k} x_{k+1}^{\gamma}
$$

where $s_{k} \geq 0$ for every $k \geq 0, \gamma>1$ is a quotient of odd natural numbers, has a positive solution satisfying $x_{0}=A>0, \lim _{k} x_{k}=x_{\infty}=0$ if

$$
\sum_{k=0}^{\infty} k s_{k+1}=\infty
$$

is satisfied. Such a result can be obtained easily by applying Corollary 2.6. Indeed it is known (see, e.g., [15, Theorem 2], [1, Theorem 6.3.4]) that the linear equation

$$
\Delta^{2} z_{k}=s_{k} u_{k+1}^{\gamma-1} z_{k+1}
$$

has a unique solution $z=T(u)$ satisfying

$$
z_{0}=A, \quad z_{k} \geq 0, \quad \Delta z_{k} \leq 0
$$

for any $u \in \Omega=\left\{u: 0 \leq u_{k} \leq A, k \geq 0\right\}$. In addition such a solution is a recessive solution of (4.3). It is immediate that $T(\Omega) \subset \Omega$. Since $B$ is closed, we have $\overline{T(\Omega)} \subset B$, and so, by applying Corollary 2.6, we obtain the existence of a solution $x$ of (4.1) such that $x_{0}=A$, $x_{k} \geq 0, \Delta x_{k} \leq 0$. Clearly $x_{k}>0$ for any $k>0$. Otherwise, if there exists $N>0$ such that $x_{k}=0$ for $k \geq N$ and $x_{N-1}>0$, from (4.1) we obtain $0=\Delta^{2} x_{N-1}=x_{N-1}$, that is, a contradiction. Finally, $x_{\infty}=0$ by virtue of (4.2). Indeed, if $x_{\infty}=\ell>0$, then $x_{k} \geq \ell$ for every $k \geq 0$, and from (4.1) we obtain $(N \geq k \geq 0)$

$$
-\Delta x_{k}=\sum_{j=k}^{N} s_{j} x_{j+1}^{\gamma}-\Delta x_{N+1} \geq \ell^{\gamma} \sum_{j=k}^{N} s_{j}
$$


which implies

$$
x_{0}-x_{k+1} \geq \ell^{\gamma} \sum_{i=0}^{k} \sum_{j=k}^{N} s_{j}=\ell^{\gamma} \sum_{j=0}^{N} s_{j}(j+1) .
$$

Letting $N \rightarrow \infty$, from (4.2) it follows that $x_{\infty}=-\infty$, a contradiction. Therefore $x_{\infty}=0$ and the result is proved.

Notice that in the applications given in Section 3, by virtue of the choice of the vector function $G$, both systems in (3.8) and in (3.25) are linear and nonhomogeneous and the conditions (b) and (c) of Theorem 2.1 are proved directly by solving (3.8), or (3.25). In the above example the choice of the function $G$ yields the linear homogeneous (4.3). Consequently, conditions (b) and (c) of Corollary 2.6 are verified by using some qualitative properties of second order linear difference equation, and not by writing explicitly the solution of (4.3)-(4.4), that would be impossible.

Finally, we point out that the above argument can be applied, with minor changes, also to treat difference equations with more general nonlinearities.

\section{Acknowledgments}

The work of the third author was supported by the Grant KJB1019407 of the Grant Agency of the Czech Academy of Sciences, the Grant G201/04/0580 of the Czech Grant Agency, and the Institutional Research Plan AV0Z010190503.

\section{References}

[1] R. P. Agarwal, Difference Equations and Inequalities. Theory, Methods, and Applications, 2nd ed., Monographs and Textbooks in Pure and Applied Mathematics, vol. 228, Marcel Dekker, New York, 2000.

[2] R. P. Agarwal and D. O'Regan, Existence and approximation of solutions of non-linear discrete systems on infinite intervals, Mathematical Methods in the Applied Sciences 22 (1999), no. 1, 91-99.

[3] _ Infinite Interval Problems for Differential, Difference and Integral Equations, Kluwer Academic, Dordrecht, 2001.

[4] _ Nonlinear Urysohn discrete equations on the infinite interval: a fixed-point approach, Computers \& Mathematics with Applications. An International Journal 42 (2001), no. 3-5, 273281.

[5] R. P. Agarwal, D. O'Regan, and P. J. Y. Wong, Positive Solutions of Differential, Difference and Integral Equations, Kluwer Academic, Dordrecht, 1999.

[6] A. Cabada, Extremal solutions for the difference $\phi$-Laplacian problem with nonlinear functional boundary conditions, Computers \& Mathematics with Applications. An International Journal 42 (2001), no. 3-5, 593-601.

[7] M. Cecchi, Z. Došlá, and M. Marini, Positive decreasing solutions of quasi-linear difference equations, Computers \& Mathematics with Applications. An International Journal 42 (2001), no. 10$11,1401-1410$.

[8] M. Cecchi, M. Furi, and M. Marini, On continuity and compactness of some nonlinear operators associated with differential equations in noncompact intervals, Nonlinear Analysis. Theory, Methods \& Applications. An International Multidisciplinary Journal. Series A: Theory and Methods 9 (1985), no. 2, 171-180. 
[9] J. R. Graef and J. Henderson, Double solutions of boundary value problems for 2 mth-order differential equations and difference equations, Computers \& Mathematics with Applications. An International Journal 45 (2003), no. 6-9, 873-885.

[10] J. R. Graef, C. Qian, and B. Yang, A three point boundary value problem for nonlinear fourth order differential equations, Journal of Mathematical Analysis and Applications 287 (2003), no. 1, 217 233.

[11] V. Marić, Regular Variation and Differential Equations, Lecture Notes in Mathematics, vol. 1726, Springer, Berlin, 2000.

[12] M. Marini, S. Matucci, and P. Řehák, Oscillation of coupled nonlinear discrete systems, Journal of Mathematical Analysis and Applications 295 (2004), no. 2, 459-472.

[13] R. Medina and M. Pinto, Convergent solutions of functional difference equations, Journal of Difference Equations and Applications 3 (1998), no. 3-4, 277-288.

[14] M. Migda, A. Musielak, and E. Schmeidel, On a class of fourth-order nonlinear difference equations, Advances in Difference Equations 2004 (2004), no. 1, 23-36.

[15] W. T. Patula, Growth and oscillation properties of second order linear difference equations, SIAM Journal on Mathematical Analysis 10 (1979), no. 1, 55-61.

[16] J. Rodriguez, Nonlinear discrete systems with global boundary conditions, Journal of Mathematical Analysis and Applications 286 (2003), no. 2, 782-794.

[17] E. Schmeidel and B. Szmanda, Oscillatory and asymptotic behavior of certain difference equation, Nonlinear Analysis. Theory, Methods \& Applications. An International Multidisciplinary Journal. Series A: Theory and Methods 47 (2001), no. 7, 4731-4742.

[18] X. Wang and L. Liao, Oscillation for even-order delay difference equations with unstable type, Applied Mathematics and Computation 153 (2004), no. 1, 289-299.

[19] J. Yan and B. Liu, Oscillatory and asymptotic behaviour of fourth order nonlinear difference equations, Acta Mathematica Sinica. New Series 13 (1997), no. 1, 105-115.

Mauro Marini: Department of Electronics and Telecommunications, University of Florence, I-50139 Florence, Italy

E-mail address: mauro.marini@unifi.it

Serena Matucci: Department of Electronics and Telecommunications, University of Florence, I-50139 Florence, Italy

E-mail address: serena.matucci@unifi.it

Pavel Řehák: Mathematical Institute, Academy of Sciences of the Czech Republic,

CZ-61662 Brno, Czech Republic

E-mail address: rehak@math.muni.cz 